\section{Benzodiazepines and Catatonia}

To the Editor:

We read with interest the recent overview on "Benzodiazepines and Catatonia" by Menza and Harris (26: 842-846, 1989). The authors noted that benzodiazepines have an anticholinergic effect (Scatton and Bartholini, 1982), which may be relevant to their utility in the treatment of catatonia. In an effort to evaluate this conclusion, we studied the effect of benztropine in the treatment of catatonic symptoms in an otherwise medication-free patient with a diagnosis of schizoaffective disurder.

The patient was a 55-year-old woman with a 36year history of episodes of psychosis and depression, separately and in cosibination. Past treatments included neuroleptic metication and electroconvulsive therapy. She had functioned well for 5 years on a low dose of thiothixene until 8 months prior to admission. Her benavior then became erratic, her speech at times illogical, and her mood labile. After stopping her medication 3 months prior to admission, her functioning declined substantially, and she was fired from three johs.

When ner neighbors noticed all of her furniture on her lawn, the police were called and found the patient sitting on her basement stairs, mute and unresponsive. She was admitted soon after.

On examination, she was immobile except for occasionally repositioning her legs. She intermittently squeezed the examiner's hand, and looked tovard the physician on command. Physical and neurological examinations were normal, although she offered occasional resistance. She tightly closed her eyes and clenched her jaws when examination of eyes and mouth was attempted. If so positioned, she would remain seated or standing for several seconds, but would gradually return to supine posture. She focused on her Image in a hand-icld mirror if held in front of her.

Laibratory work revealed a decreased potassium level $(3.2 \mathrm{mEq} / \mathrm{liter})$, but all other studies were unremarkable incluciirs complete blood count, urinalysis, drug screen, FTA, VDRL, arterial blood gases, thyroid function tests, sedimentation rate, electrocardiogram, chest radiogram, and computed tomography of the head without contrast.

The initial impression was that she was catatonic.
To assess the effects of an anticholinergic agent, wc administered, in an open design, an intramuscular injection of $2 \mathrm{mg}$ of benztropine. 'nitially, this was without effect. Two hours later we repeated the same dosage immediately after which she said, "Thank you." She gradually became more alert and communicative from that point, responded to questions appropriately (first with nods of the head, then verbally), and began eating and drinking. She was very suspicious of the staff and not fully cooperative with questioning or further laboratory work, but all this improved over the following days. She admitted she had been mildly depressed recently over the loss of employment, and that not taking her medication had been a mistake. For the remainder of her hospitalization she was remarkably pleasant although somewhat evasive, but fully cooperative. Thiothixene was resumed, and lithium carbonate was added pirophylactically. The catatonia did not recur, her suspiciousness resolved for the most part, and she was discharged in good condition 4 weeks after admission.

The rapid improvement in catatonic symptoms observed in our patient following the injection of an anticholinergic agent is consistent with Menza and Harris' conclusion that the anticholinergic activity of benzodiazepines may be relevant to their utility in the treatment of catatonic symptoms. Furthermore, Kanofsky et al. (1987) have suggested that the negative syndrome may represent an attenuated form of the catatonic syndrome. Tandon and Greden (1989) have implicated increased muscarinic activity as a possible mechanism in the production of negative schizophrenic symptoms. Interpretation must be limited because of the open, uncontrolled design, but our finding of a dramatic improvement in catatonic symptoms in a schizoaffective patient following benztropine would be consisient with this association. The utility of anticholinergic agents in the treatment of catatonic symptoms neeás to be systematically evaluated.

Michael Panzer

Rajiv Tandin

John F. Greder?

Departraent of Psychiatry

Uaiversity of Fichigati Merical Center

Ann Arbor, MI 48109-C120 


\section{References}

Kanofsky JD, Kay SR, Limdenmayer JP, Opier LA (1987): Catatonic schizophrenia and regative syndrome. Can J Psychiatry 32:162.

Scatton B, Bäribolini G (1982): GABA receptor stimulation IV. Effect of progabide (SL 76,002 ) and other GABAergic agents on acetylcholine tumover in rat brain areas. J Pharmacol Exp Ther 220:689-695.

Tanjor R, Greden JF (1959): Cholinergic hyperactivity and negative schizophrenic symptoms: $A$ model of dopaminergic/cholinergic interuckiluss in schizophrenia. Arch Gen Psychiatry 46:745-753.

\section{Response}

To the Editor:

This case report is interesting irom both practicai and theoreticai perspectives. We are unaware of any previous reports of such a response of casaionic symptoms to an anticholinergis agent and, insofar as her response can be attributed to benztropine, we agree that this may te of some clinical importance and shouid be studied more carefully.

We speculated in our paper that the usefulness of low-dose benzodiazepines in decreased dopaminergic rigid/catatonic states such as neuroleptic-induced catatonia and neuroleptic malignant syndrome may lie in their anticholinergic effects. The patient described here appears to have a more typical "psychogenic" catatonia and perhaps the anticholinergic effect of low-dose benzodiazepines also operate in some of these cases.

Tandon and Greden (1989) have suggested thast the negative symptoms of schizophrenia may resuit from a cholinergic/dopaminergic imbalance anaiogous to that seen in parkinsonian movement disor ders. If, as Kanofsky et al. (1987) suggest, there is a relationship between negative symptoms and ca- tatonia, then we might view catatonia more broadly as a ctopaminergic/cholinergic imbalance. This case report is supportive or̃ such a model.

However, as pointed cut in our paper, Pfeiffer and Jenney (1957) describe schizophrenic patients who had "lucid intervals" which included marked increases in both motor and verbal behavior in response to muscarinic cholinergic agenis. Interestingly, not all patients improved; some developed sobbing and apprehension asi they did with amobarbital. These responses would not seem to be consistent with a cholinergic hyperactivity model.

Casatonia is etiologically a heterogeneous illness and it is not surprising that different treatments work in oifferent cases. We look forward to further elucidation of the role of anticholinergic agents.

Matthew A. Menza Dale Harris

\author{
UMDNJ-Robert Wood Johnson University \\ Medical School \\ Department of Psychiatry \\ New Brunswick, New Jersey 08903
}

\section{References}

Kanorssky JD, Kay SR, Lindingayer IP, Opler LA (1987): Catatoric schizophrenia and negetive syrdome. Can J Bsychiary 32:162.

I'feiffer CC, Jenney EH (1957): The inhihition of the conditioned response and the counteraction of schizophrenia by muscarizic stimulation of the brain. Ann NY Acad Sci 66:753-764.

Tanuion R, Greden JF (i989): Cuolinergic hyperactivity and negative schizophrenic symptoms: $A$ model of dopaminergic scholinergic unteractions in schizophrenia. Arch Gen Psychiatry 46:745-753. 